Published in The Astrophysical Journal, 683, L103-L106, 2008 August 20

Preprint typeset using $\mathrm{LAT}_{\mathrm{E}} \mathrm{X}$ style emulateapj v. 08/22/09

\title{
COLOR PROFILES OF SPIRAL GALAXIES: CLUES ON OUTER-DISK FORMATION SCENARIOS
}

\author{
Judit Bakos And IGNACio TrujILlo ${ }^{1}$ \\ Instituto de Astrofísica de Canarias, E-38205, La Laguna, Tenerife, Spain; jbakos@iac.es, trujillo@iac.es \\ AND \\ Michael Pohlen \\ Cardiff University, School of Physics \& Astronomy, Cardiff, CF24 3AA, Wales, UK; Michael.Pohlen@astro.cf.ac.uk \\ Published in The Astrophysical Journal, 683, L103-L106, 2008 August 20
}

\begin{abstract}
We have explored radial color and stellar surface mass density profiles for a sample of 85 latetype spiral galaxies with deep (down to $\sim 27 \mathrm{mag} \operatorname{arcsec}{ }^{-2}$ ) SDSS $g^{\prime}$ and $r^{\prime}$ band surface brightness profiles. About $90 \%$ of the light profiles have been classified as broken exponentials, exhibiting either truncations (Type II galaxies) or antitruncations (Type III galaxies). The color profiles of Type II galaxies show a "U shape" with a minimum of $\left(g^{\prime}-r^{\prime}\right)=0.47 \pm 0.02$ mag at the break radius. Around the break radius, Type III galaxies have a plateau region with a color of $\left(g^{\prime}-r^{\prime}\right)=0.57 \pm 0.02$. Using the color to calculate the stellar surface mass density profiles reveals a surprising result. The breaks, well established in the light profiles of the truncated galaxies, are almost gone, and the mass profiles now resemble those of the pure exponential (Type I) galaxies. This result suggests that the origin of the break in Type II galaxies is more likely due to a radial change in stellar population than being associated with an actual drop in the distribution of mass. Type III galaxies, however, seem to preserve their shape in the stellar mass density profiles. We find that the stellar surface mass density at the break for truncated galaxies is $13.6 \pm 1.6 M_{\odot} \mathrm{pc}^{-2}$ and for the antitruncated ones is $9.9 \pm 1.3 M_{\odot} \mathrm{pc}^{-2}$ for the antitruncated ones. We estimate that the fraction of stellar mass outside the break radius is $\sim 15 \%$ for truncated galaxies and $\sim 9 \%$ for antitruncated galaxies.

Subject headings: galaxies: evolution - galaxies: formation - galaxies: photometry - galaxies: spiral galaxies: structure
\end{abstract}

\section{INTRODUCTION}

Our picture regarding the diversity of the radial surface brightness profiles of spiral galaxies has changed greatly since the early work of Patterson (1940) and de Vaucouleurs (1958), who showed that the disks of spiral galaxies generally follow an exponential decrease in their radial surface brightness profile. Nowadays, this view has become clearly insufficient, as not all the disk galaxies (indeed, only the minority for late-types) are well described with a single exponential fitting function as shown in several recent studies (Erwin et al. 2005; Pohlen \& Trujillo 2006, hereafter PT06; Florido et al. 2006, 2007; Erwin et al. 2008), where they have identified three basic classes of surface brightness profiles depending on an apparent break feature or lack of one: (1) the pure exponential profiles (Type I) with no breaks, (2) Type II with a "downbending break" (revising and extending a previous classification introduced by Freeman [1970] to include the so-called truncations of the stellar populations at the edge of the disk discovered by van der Kruit [1979]) and (3) a completely new class (Type III), also described by a broken exponential but with an upbending profile. The latter, discovered by Erwin et al. (2005), is also termed antitruncated.

PT06 explored a sample of nearby late-type galaxies using the Sloan Digital Sky Survey to create a statistically representative set of radial surface brightness profiles. They found that about $60 \%$ of the spirals are truncated (Type II), 30\% are antitruncated (Type III), and

\footnotetext{
${ }^{1}$ Ramón y Cajal Fellow
}

only $10 \%$ have no detectable breaks (Type I).

Still, little is known about the nature of the breaks or about the presence of stars beyond that feature. In such low-density $\left(\leq 10 M_{\odot} \mathrm{pc}^{-2}\right.$ ) environments at the galaxy peripheries, current star formation theories forbid efficient star formation (Kennicutt 1989; Elmegreen \& Parravano 1994; Schaye 2004). However, UV observations (Gil de Paz et al. 2005; Thilker et al. 2005) have shown ongoing star formation in these outer regions. In addition, it is clear that there are large number of stars in the outskirts of galaxies (see, e.g., PT06).

Several theories have investigated the formation of breaks in case of the Type II morphology. Proposed models to explain the existence of truncations in stellar disks can be grouped into two branches depending on the relevant mechanism that causes the break: (a) models related to angular momentum conservation in the protogalactic cloud (van der Kruit 1987) or angular momentum cutoff in cooling gas (van den Bosch 2001), and (b) models that attribute the existence of breaks to star formation thresholds (Kennicutt 1989; Elmegreen \& Parravano 1994; Schaye 2004). In agreement with this last scenario, Elmegreen \& Hunter (2006) suggested a multicomponent star formation that would result in a double exponential surface brightness profile as observed for Type II galaxies. Recent developments, some of them combining pieces of the aforementioned scenarios, conclude that secular evolution driven by bars or spiral arms or even clump disruptions can result in truncated exponential profiles (Debattista et al. 2006; Bournaud al. 2007; Roškar et al. 2007; Foyle et al. 2008). Magnetic 
fields have also been considered to explain the existence of truncations (Battaner et al. 2002). On the other hand, the Type III morphology is proposed to be explained by a tidal stripping within a minor merger (Peñarrubia et al. 2006; Younger et al. 2007), by a bombardment of the disk with dark matter subhalos (Kazantzidis et al. 2007) or by a high eccentricity flyby of a satellite galaxy (Younger et al. 2008).

In this Letter we show for the first time color and stellar surface mass profiles of a large sample of galaxies (the PT06 sample) to quantify the stellar mass density at the break position and the fraction of stellar mass beyond the break. These values become important while comparing observations to the results of numerical simulations for the outer-disk formation. In order to fully understand the galaxy formation and evolution process, it is necessary to perform a detailed study of the stellar population properties at the galaxies' outskirts. This kind of study gives insight into how the star formation progresses in the different parts of the disks, providing clues on the stellar mass buildup process.

\section{THE DATA AND ANALYSIS TECHNIQUES}

Our data are the 85 SDSS $g^{\prime}$ and $r^{\prime}$ band surface brightness profiles published in PT06. The galaxies were selected to be a representative, volume limited $(R \lesssim 46$ $\mathrm{Mpc}$ ) sample of face-on to intermediate-inclined late-type disk galaxies brighter than $M_{B}=-18.4 \mathrm{mag}$. In that sense, they range from fainter to brighter surface brightness, from lower to higher mass, and also from smaller to larger size. The surface brightness profiles are classified as 9 Type I, 39 Type II and 21 Type III, i.e., exponential, truncated and antitruncated profiles, respectively (see PT06 and Erwin et al. 2008 for more details).

The surface brightness limits on our individual surface brightness profiles (27.0 and $27.5 \mathrm{mag} \operatorname{arcsec}^{-2}$ for $r^{\prime}$ and $g^{\prime}$ - bands, respectively) were estimated by computing when either over- or undersubstracting the sky by $\pm 1 \sigma$ has an effect of more than 0.2 mag on the surface brightness distribution. These limits were established using two different methods for determining the sky. In addition, by comparing our profiles with deeper data (when available), we did not detect any systematic error in the sky determination. It is worth noting that, for the work presented here where we combine several profiles to explore the mean properties of the surface brightness profiles, the uncertainties in the mean properties due to sky substraction uncertainties are reduced to $\lesssim 0.03-0.04$ mag in the outermost regions of the galaxies.

We have removed 16 galaxies from our original dataset due to peculiarities of the classification, for example, all of the Type II-AB galaxies where the apparent break is to some extent artificial (see detailed discussion in PT06). We also excluded IC 1067, which has a very uncertain classification, being either Type II or a possible Type I. For galaxies with mixed classifications (see PT06) we only used the first type. To statistically compare the surface brightness profiles of all galaxies in our sample, we normalized the sizes of the different galaxies according to their respective $r^{\prime}$-band break radii (see Fig. 1). For the Type I galaxies, lacking a break radius, we applied 2.5 times the measured scale length as a normalizing factor, which is the typical radius of the break for the Type II galaxies (PT06). The Type II and Type III galaxies have their $r^{\prime}$-band $27 \mathrm{mag} \operatorname{arcsec}^{-} 2$ isophote at around 1.8 times the break radius, which constrains how far we can accurately trace out the behavior of the light profiles into the outskirts of the disks.

In order to calculate a robust mean color that characterizes our sample, we removed the color of each individual galaxy at a given radii where the $g^{\prime}$ - or the $r^{\prime}$-band surface brightness is below the above critical limits at that radii. We have obtained a robust mean value of the color for all galaxies by removing the $3 \sigma$ outliers. We have explicitly checked that using a cut in the surface brightness does not result in a bias towards any absolute magnitude range.

The results of this mean are the profiles shown in the middle row of Figure 1. It is straightforward to link the stellar mass density $(\Sigma)$ profile with the surface brightness profile at a given wavelength $\left(\mu_{\lambda}\right)$ if we know the mass-to-light $(M / L)$ ratio, using the expression below.

$$
\log _{10} \Sigma=\log _{10}(M / L)_{\lambda}-0.4\left(\mu_{\lambda}-m_{a b s, \odot, \lambda}\right)+8.629
$$

where $m_{a b s, \odot, \lambda}$ is the absolute magnitude of the Sun at wavelength $\lambda$, and $\Sigma$ is measured in $M_{\odot} \mathrm{pc}^{-2}$. To evaluate the above expression, we need to obtain the $M / L$ ratio at each radius. Following the prescription of Bell et al. (2003), we have calculated the $M / L$ ratio as a function of color.

In this work we assume a Kroupa IMF (Kroupa 2001), which according to Bell et al. (2003) implies a deduction of 0.15 dex from the $M / L$ using the following expression:

$$
\log _{10}(M / L)_{\lambda}=\left(a_{\lambda}+b_{\lambda} \times \text { color }\right)-0.15
$$

where for $\left(g^{\prime}-r^{\prime}\right)$ color, $a_{\lambda}=-0.306$ and $b_{\lambda}=1.097$ is applied to determine the $r^{\prime}$-band $M / L$. The resulting stellar mass density profiles are shown in the bottom row of Figure 1 .

The Galactic extinction has been taken into account as described in PT06 using the Schlegel et al. (1998) values:

$$
\mu_{\text {corrected }, \lambda}=\mu_{\text {measured }, \lambda}-A_{\lambda},
$$

where $A_{\lambda}$ is the extinction coefficient in each band.

\section{RESULTS}

\subsection{Averaged Radial Surface Brightness}

The upper row of Figure 1] shows the averaged radial surface brightness profiles for the Type I, Type II and Type III galaxies. The increase towards the center over the inwards extrapolated (inner) disk starting typically at around $0.2 R / R_{b}$ is due to the presence of bulges. In the outer regions the characteristic break features, truncations and antitruncations, are clearly seen in the mean profiles of Type II and III galaxies, respectively.

\subsection{Color Gradients}

The middle row of Figure 1 shows the $\left(g^{\prime}-r^{\prime}\right)$ radial color profiles. It is interesting to note that each galaxy type has its own characteristic color gradient. As found in previous works (e.g., de Jong 1996), the disks exhibit a general bluing as a function of their radius. This is seen for all the galaxy types.

The color of Type I galaxies, after reaching an asymptotic value of $\left(g^{\prime}-r^{\prime}\right) \sim 0.46 \mathrm{mag}$ in the outer regions $\left(\sim 2 R_{h}\right)$, stays within the error bars unchanged 
beyond. Type II galaxies show a minimum $\left[\right.$ at $\left(g^{\prime}-r^{\prime}\right)=$ $0.47 \pm 0.02 \mathrm{mag}]$ in their color profile at the break radius with the profile getting redder beyond. After the initial bluing, the color of Type III galaxies gets redder towards the break radius to a mean value of $\left(g^{\prime}-r^{\prime}\right)=0.57 \pm 0.02$ mag. It is important to note that we can recover the above color behavior of the mean profile basically for every individual galaxy of each subsample, so consequently this is not an artifact of our profile combination. In the outermost region of the profile, the uncertainty in the sky determination can slightly increase the error bar on the color determination. We have estimated this to be a factor of $\sim \sqrt{2}$ larger.

All galaxy types have a feature in common (see Fig. 11): a large scatter of the surface brightness and color profiles. To understand the origin of this scatter, we have explored how the color at the break position correlates with different properties of the galaxies. We find that the scatter is best correlated with the total absolute magnitude of the individual galaxies (see Fig. 2, left column). To quantify the strength of these correlations, we have performed Spearman correlation analysis. For all the three types we find that the brighter the galaxy, the redder the color at the break. This correlation is weaker for Type I galaxies, because the sample is too small to provide reliable statistics, but becomes particularly clear for Type II profiles where our $r^{\prime}$-band absolute magnitude range is the largest. Figure 2 shows that at a fixed absolute magnitude, the range of the $\left(g^{\prime}-r^{\prime}\right)$ color at the break is only $\sim 0.15$ mag. This is a factor of 2 smaller than the overall range in Figure 1 .

\subsection{Surface Mass Density Profiles}

As explained in $\S 2$, we have obtained $M / L$ ratio profiles from the $\left(g^{\prime}-r^{\prime}\right)$ colors. These profiles were then converted into stellar surface mass density profiles, which we will discuss here. The most striking result is that both Type I and Type II galaxy profiles look very similar, even without any quantitative measurement of the steepness. The break for the Type II galaxies that is so apparent in the light profiles has almost (for some individual galaxies completely) disappeared. In case of Type III galaxies, the shape of the profile has not changed dramatically: a change of the slope around the break is still evident; however, the slope becomes less well described by two individual exponentials.

\subsection{Break Surface Mass Density and Stellar Mass Fraction beyond the Break Radius}

Since the stellar surface mass density is an important tracer of star formation and disk stability (Kennicutt 1989), we provide here numbers corresponding to the break position. The middle column of Figure 2 shows the histograms of stellar surface mass density at the break radius or, for Type I galaxies, at $R=2.5 R_{h}$. The values of $\Sigma_{b r}$ are $22.5 \pm 5.3 M_{\odot} \mathrm{pc}^{-2}$ (Type I), $13.6 \pm 1.6 M_{\odot} \mathrm{pc}^{-2}$ (Type II), and $9.9 \pm 1.3 M_{\odot} \mathrm{pc}^{-2}$ (Type III). Note that we do not detect any brek on the stellar mass density profile of Type I galaxies down to $\sim 3 M_{\odot} \mathrm{pc}^{-2}$

Another quantity we have calculated is the amount of stellar mass beyond the break radii, which can be very useful for constraining the theoretical model. In the right column of Figure 2, the stellar mass fractions in the outer disk are shown. Type I galaxies contain about $22.3 \pm 2 \%$ of their total stellar mass beyond a radius of 2.5 times their scale-length. Type II and Type III galaxies have much less stellar mass in the outer regions. For Type III galaxies $(9.2 \pm 1.4 \%)$ the amount of stellar mass is the lowest, with Type II in between $(14.7 \pm 1.2 \%)$.

\section{DISCUSSION}

How can the results found in this work be used to constrain the current models for the formation of breaks in the surface brightness profiles of disk galaxies?

In the case of Type II galaxies, the traditional pictures of break formation (see $\S$ 1) grouped into two families: angular momentum versus thresholds of star formation. Neither of these two ideas taken at face value can explain why we find so many stars beyond the break radius, so we will not go into more detail on these individual models. However, it is important to stress that the new rendition of models has been able to naturally explain the existence of stars beyond the break radius, and even more, the exponential nature of the shape of the surface brightness beyond this feature. In particular, in the case of the Roškar et al. (2008) model in which the breaks are the result of the interplay between a radial star formation cutoff and a redistribution of stellar mass by secular processes, a natural prediction is the existence of a minimum in the age of the stellar population at the break position, and a further aging (and consequently, a likely reddening) of these stars as we move farther and farther away from the break radius. This is in qualitative agreement with what we see in our color profiles for this kind of galaxy. However, what these models have not been able to reproduce is the absence of a clear break in the stellar mass density profile.

The near absence of a break in the stellar surface mass density profile for our galaxies gives a strong indication that the behavior of the surface brightness profile outside the break is basically due to a change in the ingredients of the stellar population. If the shape of the color we see is not caused by a change in metallicity, this behavior could be explained as a natural consequence of stochastic migration of young stars from the inner parts of the disk to the outskirts (Roškar et al. 2008). This will result in an age gradient where the oldest stars are the dominant component in the outskirts of the disks.

Unfortunately, other models (like Bournaud et al. 2007 and Foyle et al. 2008) that are capable of creating stellar mass density profiles that resemble the Type II one we have found here (i.e., without a clear break) do not provide any prediction on the color (age) distribution the stars should have along the radial range.

Nevertheless, the fact that the stellar mass density of the break for this type of galaxy with $\sim 13 M_{\odot} \mathrm{pc}^{-2}$ is so close to the gas density threshold prediction of $\sim 10$ $M_{\odot} \mathrm{pc}^{-2}$ makes the case for a stellar population origin for the surface brightness break even stronger (with a $100 \%$ efficiency of transforming gas to stars).

Evidence for the same color phenomenology for Type II galaxies also at high redshift is presented by Azzollini et al. (2008). They have shown that a similar minimum in the color profile can be found, at least, up to $\mathrm{z} \sim 1$ and that the main source of the scatter of the color profiles is caused by the different stellar mass (in our case, absolute magnitude) of the galaxies in their sample. Following 
these findings, we can conclude that once the absolute magnitude of a galaxy is fixed, the color profiles within a given Type (I, II or III) of galaxy are strikingly similar. A more massive galaxy has a redder global color but the same shape of the color gradient.

Combining the results found in Azzollini et al. (2008) with what we find here, one is tempted to claim that both the existence of the break in Type II galaxies and the shape of their color profiles are long-lived features in the galaxy evolution, because it would be hard to imagine how the above features could be continuously destroyed and recreated maintaining the same properties over the last $\sim 8$ Gyr.

In the case of Type III galaxies, the situation is less clear. On the one hand, our sample is smaller than in the case of Type II galaxies and consequently our results less robust. And on the other hand, the theoretical models are less elaborated than for truncated galaxies, and no clear predictions have been made in particular for the color profiles. Taking into account that the shape of the stellar mass density profile does not differ too much from what we see in the surface brightness profiles, we are inclined to think that our data do not favor a sole origin in stellar population changes for this type of galaxy but an authentic change in the amount of stars from the exponential continuation of their inner region. It is interesting to note that in most of the proposed ideas summarized in $\S 1$ to explain this kind of galaxy, the origin of the stars in the periphery are linked to a dynamical (in some case external) origin. So what we are seeing for these galaxies is maybe a combination of star formation combined with an infall of new stars from a external (satellite) source.

To corroborate our results, we plan to increase the number of galaxies as well as the number of observed filters in our next study.

This research was supported by the Instituto Astrofísica de Canarias. We thank Alexande Vazdekis and Ruymán Azzollini for their valuable comments and the anonymous referee for his or her careful reading.

\section{REFERENCES}

Azzollini, R., Trujillo, I, Beckman, J., 2008, ApJ, 679, L69

Battaner, E., Florido, E., \& Jiménez-Vicente, J. 2002, A\&A, 388, 213

Bell, E.F., McIntosh, D.H., Katz, N., Weinberg, M.D., 2003, ApJS, 149, 289

Bournaud, F., Elmegreen, B. G., \& Elmegreen, D. M., 2007, ApJ, 670,237

Debattista, V. P., Mayer, L., Carollo, C. M., Moore, B., Wadsley, J., \& Quinn, T., 2006, ApJ, 645, 209

de Jong, R. S. 1996, A\&A, 313, 45

de Vaucouleurs, G. 1958, ApJ, 128, 465

Elmegreen, B. G. \& Hunter, D. A., 2006, ApJ, 363, 712

Elmegreen, B. G. \& Parravano, A., 1994, ApJ, 435, L121

Erwin, P., Beckman, J. E., \& Pohlen, M., 2005, ApJ, 626, L81

Erwin, P., Pohlen, M., \& Beckman, J. E., 2008, AJ, 135, 20

Florido, E., Battaner, E., Guijarro, A., Garzón, F., \& Castolli-Morales, A. 2006, A\&A, 455, 467

Florido, E., Battaner, E., Zurita, A., \& Guijarro, A. 2007, A\&A, 472, L39

Foyle, K., Courteau, S., Thacker, R. J., 2008, MNRAS, 386, 1821

Freeman, K. C. 1970, ApJ, 160, 811

Gil de Paz, A., et al. 2005, ApJ, 627, L29
Kazantzidis, S., Bullock, J. S., Zentner A. R., Kravtsov, A. V., \& Moustakas, L. A., 2007, ApJ, submitted (arXiv: 0708.1949)

Kennicutt, R. C., 1989, ApJ, 344, 685

Kroupa, P., 2001, MNRAS, 322, 231

Patterson, F. S., 1940, Harvard Coll. Obs. Bull., 914, 9

Peñarrubia, J., McConnachie, A., \& Babul, A., 2006, ApJ, 650, L33

Pohlen, M., \& Trujillo, I., 2006, A\&A, 454, 759 (PT06)

Roškar, R., Debattista, V. P., Stinson, G. S., Quinn, T. R.,

Kaufmann, T., Wadsley J., 2008, ApJ, 675, L65

Schaye, J., 2004, ApJ, 609, 667

Schlegel, D. J., Finkbeiner, D. P., Davis, M., 1998, ApJ, 500, 525

Thilker D. A., et al., 2005, ApJ, 619, L79

van den Bosch, F. C., 2001, MNRAS, 327, 1334

van der Kruit, P. C., 1979, A\&AS, 38, 15

van der Kruit, P. C., 1987, A\&A, 173, 59

Younger, J. D., Besla, G., Cox, T. J., Hernquist, L., Robertson, B., \& Willman, B., 2008, ApJ, 676, L21

Younger, J. D., Cox, T. J., Seth A. C., \& Hernquist, L., 2007, ApJ, 670, 269 


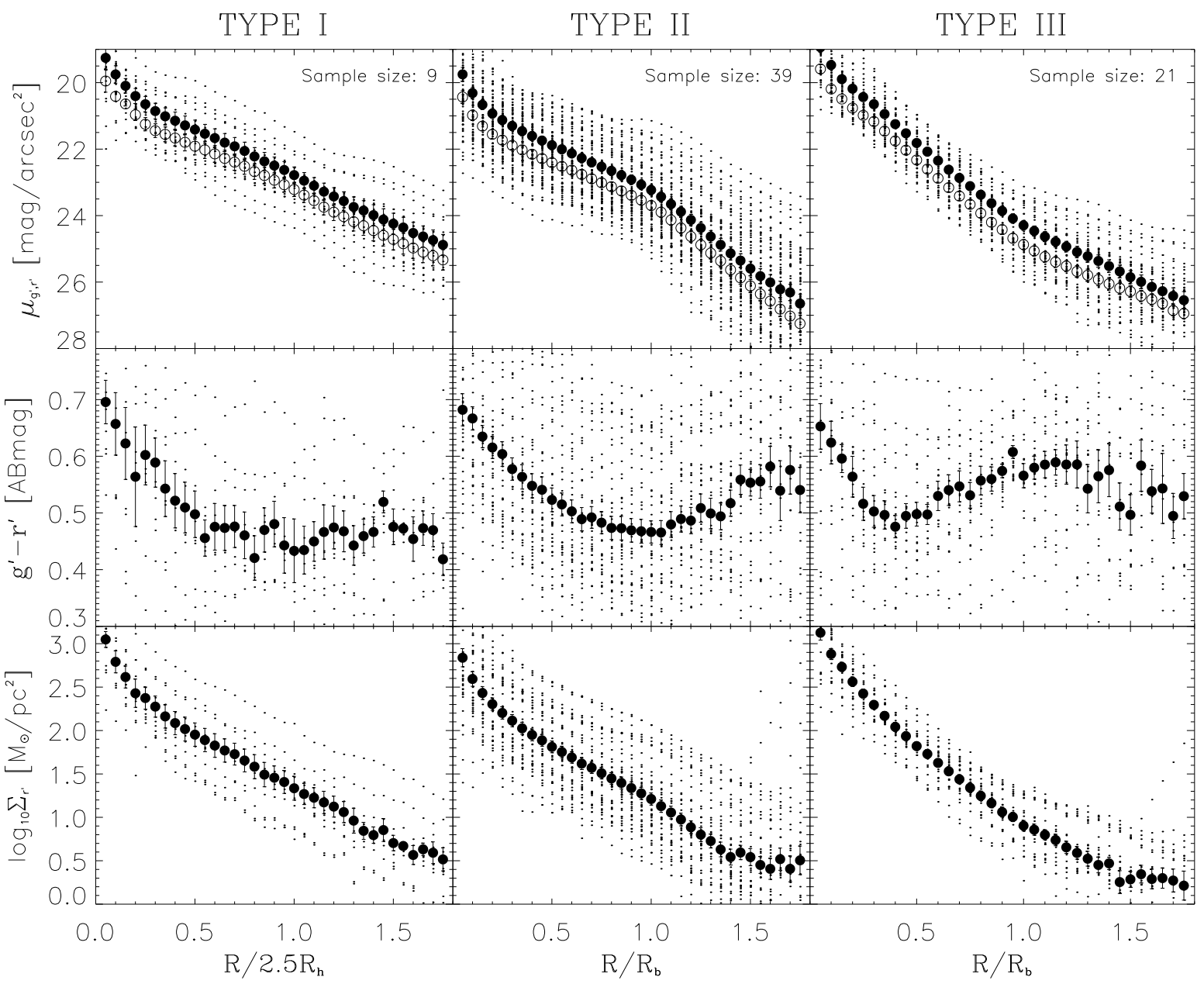

FIG. 1.- Top row: Averaged, scaled radial surface brightness profiles of 9 Type I, 39 Type II and 21 Type III galaxies. The filled circles correspond to the $r^{\prime}$-band mean surface brightness, the open circles to the mean $g^{\prime}$-band data. The small dots are the individual galaxy profiles in both bands. The surface brightness is corrected for Galactic extinction. Middle row: $\left(g^{\prime}-r^{\prime}\right)$ color gradients. The averaged profile of Type I reaches an asymptotic color value of $\sim 0.46$ mag being rather constant outward. Type II profiles have a minimum color of $0.47 \pm 0.02 \mathrm{mag}$ at the break position. The Type III mean color profile has a redder value of about $0.57 \pm 0.02 \mathrm{mag}$ at the break. Bottom row: $r^{\prime}$-band surface mass density profiles obtained using the color-to-M/L conversion of Bell et al. (2003). Note how the significance of the break almost disappears for the Type II case. The error bars are given as $\sim \sigma / \sqrt{N}$, where $\sigma$ is the scatter and $N$ is the number of galaxies taken into account for estimating the mean averaged value in each bin. These error bars do not account for uncertainties in the sky determination. 

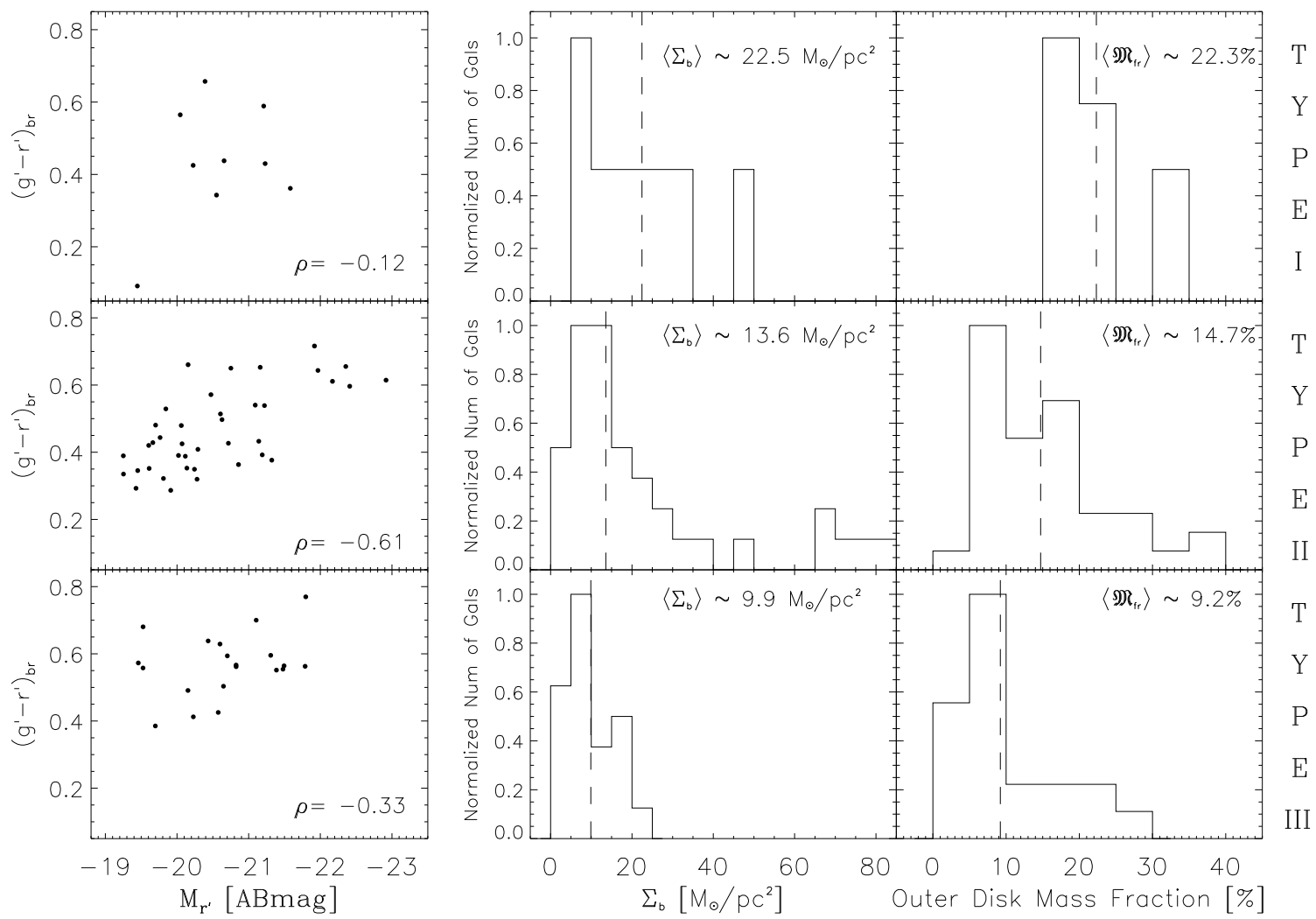

FIG. 2.- Left column: Absolute $r^{\prime}$ magnitude and break color (or at 2.5 scale lengths in the case of Type I galaxies) correlations; $\rho$ is the Spearman's correlation coefficient. Type II galaxies show a strong correlation between the break color and the absolute magnitude, which means that the scatter in break color at a given luminosity is significantly smaller than the overall scatter. Middle and right columns: Stellar surface mass density and stellar mass fraction histograms with their median values in the upper right corner of each panel. 